\title{
Molecular dynamics simulation reveals how phosphorylation of tyrosine 26 of phosphoglycerate mutase 1 upregulates glycolysis and promotes tumor growth
}

\author{
Yan Wang ${ }^{1}$, Wen-Sheng Cai ${ }^{2}$, Luonan Chen ${ }^{3}$, Guanyu Wang ${ }^{1}$ \\ ${ }^{1}$ Department of Biology, Southern University of Science and Technology, Shenzhen 518055, China \\ ${ }^{2}$ Research Center for Analytical Sciences, College of Chemistry, Nankai University, Tianjin 300071, China \\ ${ }^{3}$ Key Laboratory of Systems Biology, CAS Center for Excellence in Molecular Cell Science, Innovation Center for Cell Signaling \\ Network, Shanghai Institute of Biochemistry and Cell Biology, Chinese Academy of Sciences, University of Chinese Academy \\ of Sciences, Shanghai 200031, China \\ Correspondence to: Luonan Chen, email: Inchen@sibs.ac.cn \\ Guanyu Wang, email: wanggy@sustc.edu.cn \\ Keywords: oncotarget, glycolysis, molecular dynamics simulation \\ Received: June 29, $2016 \quad$ Accepted: December 16, 2016 \\ Published: January 05, 2017
}

\section{ABSTRACT}

\begin{abstract}
Phosphoglycerate mutase 1 (PGAM1) catalyzes the eighth step of glycolysis and is often found upregulated in cancer cells. To test the hypothesis that the phosphorylation of tyrosine 26 residue of PGAM1 greatly enhances its activity, we performed both conventional and steered molecular dynamics simulations on the binding and unbinding of PGAM1 to its substrates, with tyrosine 26 either phosphorylated or not. We analyzed the simulated data in terms of structural stability, hydrogen bond formation, binding free energy, etc. We found that tyrosine 26 phosphorylation enhances the binding of PGAM1 to its substrates through generating electrostatic environment and structural features that are advantageous to the binding. Our results may provide valuable insights into computer-aided design of drugs that specifically target cancer cells with PGAM1 tyrosine 26 phosphorylated.
\end{abstract}

\section{INTRODUCTION}

Glycolysis is a pathway that converts one molecule of glucose to two molecules of pyruvate, with the concomitant generation of two molecules of adenosine triphosphate (ATP). The latter is known as the energy currency for the cell, because it provides energy for virtually any biological processes such as biosynthesis of macromolecules. Glycolysis is of critical importance, because it bases all the other pathways of glucose metabolism; and unlike the other pathways, it requires no participation of oxygen, thus conferring survival advantages for those rapidly dividing cells such as cancer cells, which are often in the hypoxic state. Even when oxygen is abundant, cancer cells tend to avoid oxygen dependent glucose metabolism by upregulating glycolysis, a phenomenon long known as the Warburg effect [1]. The Warburg effect implies that glycolysis may possess some advantages $[1,2]$. Therefore, studying glycolysis may provide valuable insights into our understanding of cancer metabolism. Glycolysis consists of ten steps.
Phosphoglycerate mutase 1 (PGAM1) is an enzyme catalyzing the eighth step of glycolysis, namely isomerization of the substrate 3-phosphoglycerate (3PG) into 2-phosphoglycerate (2PG) (Figure 1). 3PG and 2PG differ only in the location of the phosphoryl group, which is at position C-3 in $3 \mathrm{PG}$ and $\mathrm{C}-2$ in $2 \mathrm{PG}$. The isomerization starts with an active PGAM1, namely PGAM1 with its histidine 11 residue (H11) phosphorylated. The isomerization can be divided into two half reactions $[3,4]$. First, PGAM1 binds with the substrate $3 \mathrm{PG}$ and transfers its phosphoryl group to $3 \mathrm{PG}$ at position $\mathrm{C}-2$, generating the intermediate 2,3-bisphosphoglycerate (2,3-BPG). Second, the phosphoryl group at C-3 of 2,3-BPG is transferred to H11 of PGAM1, whereby 2,3-BPG turns into the product 2PG. The phosphorylation of $\mathrm{H} 11$ reactivates PGAM1, which can catalyze a new round of isomerization.

Upregulation of PGAM1 is implicated in the development of many cancers, including hepatocellular carcinoma and colorectal cancer $[5,6]$. Rapid growth and division of cancer cells depend critically on their capability of coordinating glycolysis and biosynthesis, in which PGAM1 plays a crucial role. Hitosugi et al. found 
that PGAM1 contributes to the coordination by controlling intracellular levels of its substrate $3 \mathrm{PG}$ and product $2 \mathrm{PG}$ [7]. There exist two mechanisms to explain PGAM1 upregulation. First, the loss of TP53 leads to increased expression of $P G A M 1$, which is a negative transcriptional target of TP53 [8-10]. The second putative mechanism is the phosphorylation of tyrosine 26 (Y26) residue of PGAM1, which may greatly enhance PGAM1 activity and can explain the finding that Y26 phosphorylation is commonly found in human cancers [2]. To understand how Y26 phosphorylation may enhance PGAM1 activity, Hitosugi et al. crystalized human PGAM1 proteins with both phosphorylated and dephosphorylated H11 [2]. Comparison of the two structures suggested that Y26 phosphorylation causes a conformational change that is characterized by the leaving of the negatively charged glutamic acid 19 (E19) residue from the active site, thus promoting 2,3-BPG binding and consequently $\mathrm{H} 11$ phosphorylation. This may also help to keep the active site open for substrate (3PG) binding. In brief, the active site of PGAM1 may be partially blocked by E19 when Y26 is not phosphorylated; and Y26 phosphorylation may clear the blockage and thus enhance PGAM1 activity. Although reasonable, the explanation was based on static data and lacked dynamical evidences, including computer simulations of atomic movements whereby the enzyme-substrate binding is achieved. Indeed, the crystal structures were only two snapshots containing no such dynamical information.

In the present paper, we used molecular dynamics (MD) simulation to test the hypothesis that Y26phosphorylation enhances the activity of PGAM1 and to learn how the enhancement is achieved. MD simulation is a computational method for studying the physical movements of atoms and molecules, which are allowed to interact for a fixed period of time, giving a dynamical evolution of the system [11-14]. It is very useful in exploring enzyme-substrate interactions [15].

We first built in silico the complex 2,3-BPG:PGAM1, formed by binding of 2,3-BPG to PGAM1. The complex is called the wild type system or the Y26-phospho system, when Y26 is dephosphorylated or phosphorylated, respectively. In the following, we also use a suffix wt or phos to signify Y26's phosphorylation state. For example, PGAM1 phos represents PGAM1 with Y26 phosphorylated. We then performed MD simulations on both systems to obtain the detailed atomic movements that facilitate the binding of 2,3-BPG to PGAM1 and then calculated the binding free energy. We also studied the binding of PGAM1 to $3 \mathrm{PG}$ and $2 \mathrm{PG}$, respectively. By comparing the results, we explained how Y26 phosphorylation enhances PGAM1 activity and glycolysis.

These results may provide further insights into tumor growth and lead to drug targets for cancer by e.g. preventing Y26 phosphorylation.

\section{RESULTS \& DISCUSSION}

\section{General structural features of 2,3-BPG:PGAM1}

For each of the two systems, 900 ns MD simulation was carried out, which generated 450000 frames of trajectory data. To assess the system's structural stability, we calculated RMSD of C- $\alpha$ atoms of the protein backbone, by using the software AmberTools 15 . As shown in Figure 2A, both systems underwent fierce conformational changes during the first 300 ns, with the wild type system changing greater. Notably, the RMSD values of the wild type system exceeded $2.5 \AA$ several times, while those of the Y26-phospho system were all below $2.5 \AA$. From $300 \mathrm{~ns}$ to $700 \mathrm{~ns}$, the RMSD values of both systems fluctuated around $2.0 \AA$, with the wild type system having a slightly larger magnitude. Fluctuations reduced greatly during the last $200 \mathrm{~ns}$, with the average RMSD value reaching $\sim 1.5 \AA$ in both systems. The wild type system still fluctuated slightly more intensive than the Y26-phospho system. In line with the RMSD results, RMSF analysis also demonstrated the greater conformational variations of the wild type system (Figure 2B), indicating that residues of the wild type system are generally more flexible than those of the Y26-phospho

\section{3-PG + P-Enzyme $\longrightarrow$ 2,3-BPG + Enzyme $\longrightarrow$ 2-PG + P-Enzyme}<smiles>O=C([O-])C(O)COP(=O)([O-])[O-]</smiles>

3-phosphoglycerate (3PG)<smiles>O=C([O-])C(COP(=O)([O-])[O-])OP(=O)([O-])[O-]</smiles>

2,3-bisphosphoglycerate (2,3-BPG)<smiles>O=C([O-])C(CO)OP(=O)([O-])[O-]</smiles>

2-phosphoglycerate (2-PG)

Figure 1: The phosphoglycerate mutase reaction and the chemical structural formula of 3PG, 2,3-BPG, and 2PG. 
system. The flexibility difference was most dramatic at residues spanning different regions of PGAM1: Leucine 18 (L18), Glutamic acid 102 (E102), Alanine 105 (A105), and Aspartic acid 148 (D148). Therefore, both RMSD and RMSF analyses suggested that Y26 phosphorylation stabilizes the binding of 2,3-BPG.

The scenario may be different during periods far away from stabilization of binding. During the entry into or exit from the binding site, the enzyme may undergo significant conformational changes; and events such as mutation and single residue phosphorylation (the present case) increase the likelihood of their occurrence [16-26]. To observe such conformational changes, one has to wait a long time until the ligand egresses from the binding site, if the conventional Molecular Dynamics (cMD) simulation is used. We thus used Adaptive Steered Molecular Dynamics
(ASMD) to accelerate the simulation, with the RMSF results presented in Supplementary Figure 4 (red) against the original cMD RMSF data (black). For the wild type system, ASMD and cMD yielded close RMSF results. The most dramatic changes occurred at residues 125 to 140 , which were not significantly involved in binding. For the Y26-phospho system, RMSF values increased markedly of residues K113 to R117, which were all prominent binding residues. These data imply that Y26 phosphorylation may facilitate the initial binding of 2,3-BPG to PGAM1 by making the binding pocket open wider, namely by inducing greater conformational changes of the binding pocket.

The flexibility difference can be explained by other techniques of trajectory analysis, such as the following hydrogen bond analysis.
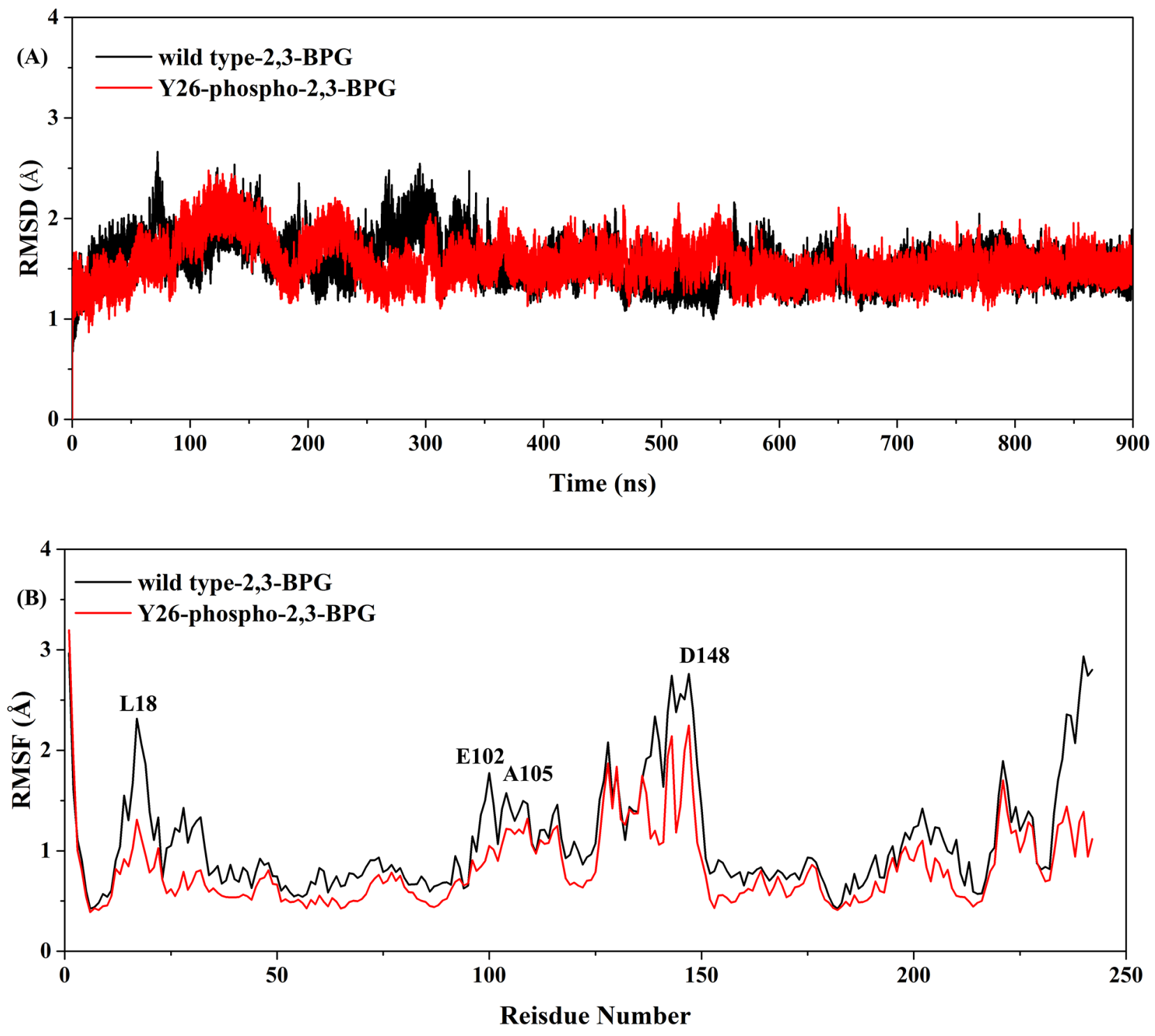

Figure 2: A. The time course of RMSD values of the wild type system (black) and the Y26-phospho system (red). B. The time course of RMSF values of the two systems. Those residues with high RMSF values are labeled. 


\section{Hydrogen bond formation in the Y26-phospho system stabilized 2,3-BPG binding}

Non-bonded interactions such as hydrogen bond, hydrophobic interaction, salt bridge play important roles in macromolecules' structural formation and biological functions. We examined the formation of hydrogen bonds between 2,3-BPG and its surrounding residues of PGAM1 over the $900 \mathrm{~ns}$ simulation for both the wild type system and the Y26-phospho system, with the results presented in Table 1 and Figure 3. Hydrogen bond formation was based on the two criteria presented in the Method section. We found that the wild type and Y26-phospho systems had 18 and 33 hydrogen bonds formed, respectively. That is, 2,3-BPG molecule in the Y26-phospho system formed more hydrogen bonds than in the wild type system. More importantly, the hydrogen bonds persisted much longer in the Y26-phospho system than in the wild type system, indicating that PGAM1 phos interacts more actively with 2,3-BPG, than PGAM1 wt interacts with 2,3-BPG.

The hydrogen bond between 2,3-BPG and Serine 23 (S23) (presented as 244@O15-S23@HG in Table 1) is worth noting. It had a $98.56 \%$ occupancy in the Y26phospho system, but was completely absent in the wild type system. The striking difference implies that Y26 played a critical role in stabilizing 244@O15-S23@HG. Given that Y26 is spatially close to S23, this is quite possible. Indeed, Y26 formed three hydrogen bonds with N135 (Table 2 and Figure 4D). These hydrogen bonds might keep S23 in an orientation that is suitable for forming 244@O15-S23@HG. The three hydrogen bonds were absent in the wild type system (Figure 4C).

Besides S23, other residues such as Lysine 100 (K100) and Asparagine 17 (N17) also formed multiple stable hydrogen bonds with 2,3-BPG in the Y26-phospho system (Table 1(b)). A previous experimental study had also revealed the importance of K100 in 2,3-BPG binding [27]. Although K100 and N17 also mediated hydrogen bond formation in the wild type system, these hydrogen bonds had much smaller occupancy and were thus unstable (Table 1(a)). These data can explain the above RMSF analysis, which demonstrated that some residues (e.g., L18, E102, A105, D148) became less flexible upon Y26 phosphorylation (Figure 2B). For example, L18 is a neighbor of N17; thus hydrogen bonds formed by N17 would fix L18 as well, making the RMSF value at L18 greatly reduced. Similarly, the reduced flexibility of E102 and A105 might be due to the hydrogen bond formed by K100.

\section{Identification of an $\alpha$-helix crucial for 2,3-BPG binding}

To obtain a comprehensive understanding of structural variances of 2,3-BPG:PGAM1, snapshots of the trajectory data were clustered by using the average linkage algorithm; and five clusters were yielded respectively for each of the two systems. We then picked five representative structures $S_{i}(i=0,1,2,3,4)$ from the five clusters, respectively. Note that $S_{0}$ was fixed to be the initial structure. For the wild type system, we found that $S_{0}$ was the largest cluster, containing $48.7 \%$ of the total snapshots. The structure $S_{0} w t$ deviated from the crystal structure with a RMSD value $1.25 \AA$. For the Y26 phospho system, we found that $S_{1}$ was the largest cluster, containing $89.2 \%$ of the total snapshots. The structure $S_{1}$ phos deviated from the crystal structure with a RMSD value $0.65 \AA$.

We then used the crystal structure (PDB ID: 3FDZ) as the reference to study the structural deviations of $S_{0} w t$ and $S_{1}$ phos, because the crystal structure had recorded faithfully the actual binding of 2,3-BPG with PGAM1. The three systems were first superimposed (Figure 5). As expected, the overall structures of the three systems were quite similar. The area around the 2,3-BPG binding site showed relatively great variances, including an $\alpha$-helix formed by residues Asparagine 99 to Glycine 108 (Figure $6)$. The $\alpha$-helix was of particular importance, because one of its constituents, K100, had been shown important for the binding of 2,3-BPG [27]. In accordance with that, we also found that K100 formed hydrogen bonds of high occupancy with 2,3-BPG in the Y26-phospho system but not in the wild type system (Table 1). The $\alpha$-helix thus deserved a more detailed analysis.

We first measured the $\alpha$-helix's center of mass for all the three structures. We then calculated the mass center deviation (relative to the crystal structure) for both $S_{0} w t$ and $S_{1}$ phos, which were $3.62 A$ and $1.38 \AA$, respectively. This result indicated that the $\alpha$-helix of the Y26-phospho system was less deviated from the actual binding position than the wild type system (Figure 5). More insights can be obtained if we study the motion of the whole system, particularly the $\alpha$-helix region. To that end, we applied principal component analysis (PCA) to both systems. For each system, we identified two principal components, which together represent more than $50 \%$ of the overall movements. The direction of movement corresponding to the first principal component is indicated by the conic arrows in Figure 6, which shows vividly the radical difference between the two systems: the $\alpha$-helix moves away from the binding site in the wild type system; while it moves towards the binding site in the Y26-phospho system. The latter movement might bring K100 to the proximity of 2,3-BPG, facilitate the formation of stable hydrogen bonds, and ultimately confer a tight binding of 2,3-BPG to PGAM1.

\section{2,3-BPG binding was an energy favorable event in the Y26-phospho system but not in the wild type system}

Affinity of enzyme-substrate binding can be estimated by calculating the free energy of binding $\Delta G_{\text {bind }}$, 
Table 1: Properties of the formed hydrogen bonds between 2,3-BPG and its adjacent PGAM1 residues (a) the wild type system

\begin{tabular}{|c|c|c|c|c|}
\hline$\#$ & H-bond & Occupancy (\%) & Distance $(\AA)$ & Angle ( $\left.{ }^{\circ}\right)$ \\
\hline 1 & 244@O7-Y92@HH & 10.82 & 2.68 & 161.78 \\
\hline 2 & 244@011-N209@HD21 & 8.51 & 2.79 & 164.14 \\
\hline 3 & 244@O8-Y92@HH & 6.26 & 2.66 & 159.88 \\
\hline 4 & 244@O9-N209@HD21 & 10.24 & 2.78 & 161.64 \\
\hline 5 & 244@O7-N188@HD21 & 5.41 & 2.83 & 157.35 \\
\hline 6 & 244@O13-N17@HD21 & 9.50 & 2.78 & 155.22 \\
\hline 7 & 244@013-K100@HZ1 & 8.17 & 2.80 & 151.06 \\
\hline 8 & 244@013-K100@HZ3 & 7.92 & 2.79 & 151.84 \\
\hline 9 & 244@O15-N17@HD21 & 6.44 & 2.81 & 157.34 \\
\hline 10 & 244@O13-K100@HZ2 & 7.32 & 2.79 & 150.19 \\
\hline 11 & 244@O14-K100@HZ1 & 9.09 & 2.79 & 149.13 \\
\hline 12 & 244@O14-K100@HZ2 & 8.10 & 2.79 & 149.42 \\
\hline 13 & 244@O15-K100@HZ1 & 9.94 & 2.80 & 151.00 \\
\hline 14 & 244@O8-N188@HD21 & 2.46 & 2.83 & 156.80 \\
\hline 15 & 244@014-K100@HZ3 & 7.74 & 2.79 & 148.44 \\
\hline 16 & 244@O15-K100@HZ2 & 9.31 & 2.80 & 152.45 \\
\hline 17 & 244@015-K100@HZ3 & 8.74 & 2.79 & 151.17 \\
\hline 18 & 244@O14-N17@HD21 & 6.10 & 2.82 & 146.39 \\
\hline
\end{tabular}

\begin{tabular}{|c|c|c|c|c|}
\hline$\#$ & H-bond & Occupancy (\%) & Distance $(\AA ̊)$ & Angle ( $\left.{ }^{\circ}\right)$ \\
\hline 1 & 244@O15-S23@HG & 98.56 & 2.58 & 165.89 \\
\hline 2 & 244@O8-Y92@HD21 & 12.51 & 2.65 & 166.63 \\
\hline 3 & 244@O13-N17@HD21 & 85.67 & 2.75 & 147.20 \\
\hline 4 & 244@O14-N17@HD21 & 77.63 & 2.89 & 145.58 \\
\hline 5 & 244@07-Y92@HH & 7.18 & 2.67 & 162.81 \\
\hline 6 & 244@014-S23@HG & 6.31 & 2.60 & 165.97 \\
\hline 7 & 244@O13-K100@HZ3 & 7.74 & 2.73 & 155.89 \\
\hline 8 & 244@O13-K100@HZ2 & 8.06 & 2.72 & 156.53 \\
\hline 9 & 244@O8-N188@HD21 & 5.90 & 2.84 & 155.37 \\
\hline 10 & 244@O9-K100@HZ1 & 5.89 & 2.76 & 157.02 \\
\hline 11 & 244@O7-N188@HD21 & 5.86 & 2.84 & 150.15 \\
\hline 12 & 244@O13-K100@HZ1 & 8.37 & 2.73 & 155.90 \\
\hline 13 & 244@O9-K100@HZ2 & 11.10 & 2.76 & 153.49 \\
\hline 14 & 244@O9-K100@HZ3 & 11.25 & 2.76 & 157.19 \\
\hline 15 & 244@013-S23@HG & 3.08 & 2.60 & 166.03 \\
\hline 16 & 244@O15-K100@HZ3 & 5.61 & 2.78 & 158.72 \\
\hline 17 & 244@O15-K100@HZ2 & 5.23 & 2.77 & 157.81 \\
\hline 18 & 244@O11-K100@HZ3 & 2.41 & 2.82 & 142.57 \\
\hline 19 & 244@O11-K100@HZ1 & 2.26 & 2.83 & 140.79 \\
\hline 20 & 244@O15-K100@HZ1 & 5.51 & 2.78 & 159.17 \\
\hline 21 & 244@O11-K100@HZ2 & 1.99 & 2.82 & 142.97 \\
\hline 22 & 244@O14-K100@HZ2 & 4.40 & 2.77 & 159.63 \\
\hline
\end{tabular}




\begin{tabular}{llcll}
23 & $244 @ O 14-K 100 @ H Z 1$ & 5.12 & 2.77 & 159.91 \\
24 & $244 @ O 15-N 17 @ H D 21$ & 77.32 & 2.69 & 145.96 \\
25 & $244 @ O 11-N 17 @ H D 22$ & 3.76 & 2.79 & 153.11 \\
26 & $244 @ O 14-K 100 @ H Z 3$ & 2.67 & 2.77 & 159.92 \\
27 & $244 @ O 7-N 209 @ H D 21$ & 1.69 & 2.81 & 157.85 \\
28 & $244 @ O 8-K 100 @ H Z 1$ & 5.52 & 2.76 & 155.11 \\
29 & $244 @ O 8-K 100 @ H Z 2$ & 5.08 & 2.76 & 155.12 \\
30 & $244 @ O 8-K 100 @ H Z 3$ & 4.95 & 2.76 & 155.14 \\
31 & $244 @ O 7-K 100 @ H Z 1$ & 4.17 & 2.76 & 154.74 \\
32 & $244 @ O 7-K 100 @ H Z 2$ & 3.80 & 2.77 & 153.48 \\
33 & $244 @ O 7-K 100 @ H Z 3$ & 3.73 & 2.76 & 154.29 \\
\hline
\end{tabular}
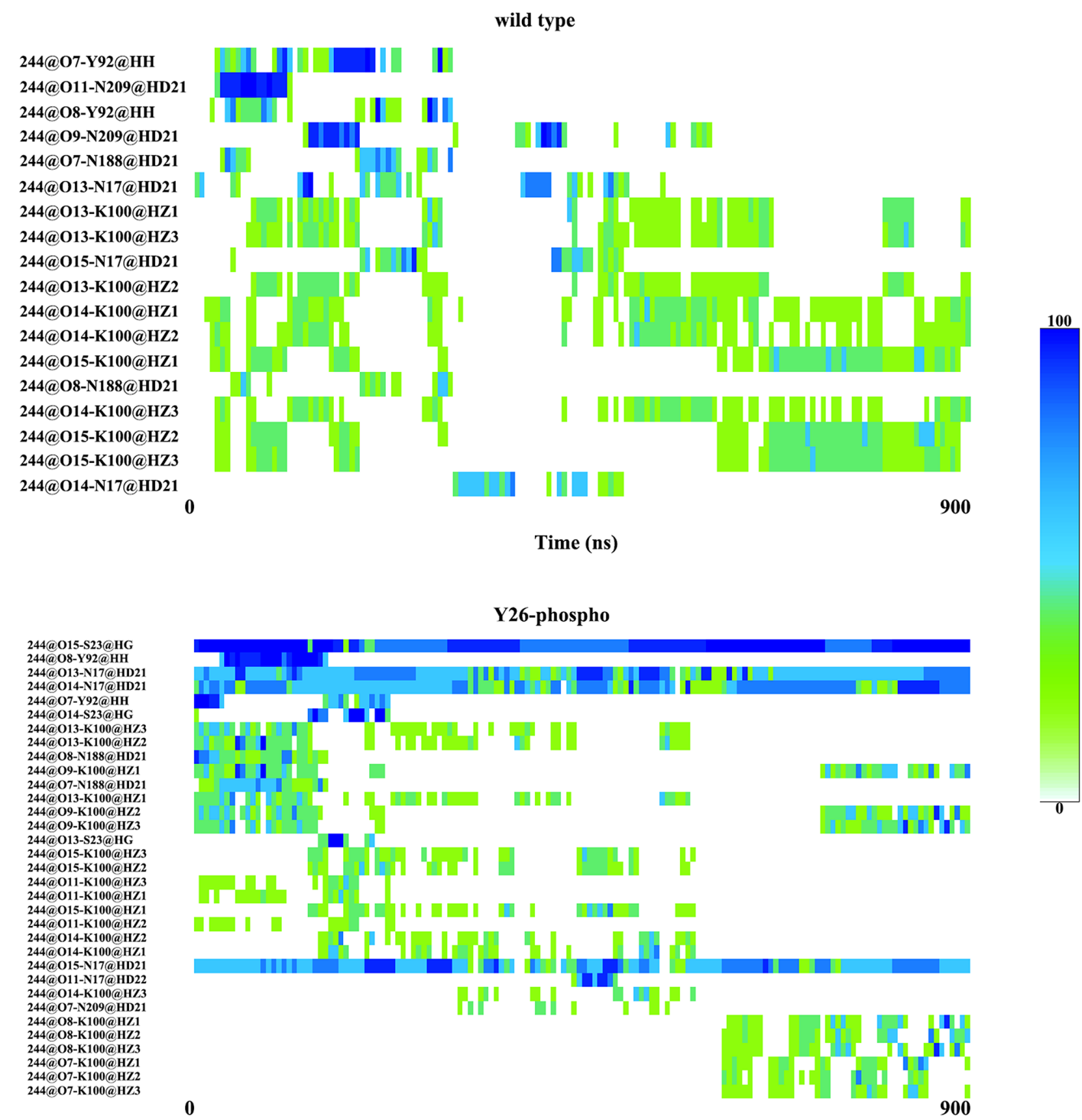

Time (ns)

Figure 3: Frequency of hydrogen bond formation between 2,3-BPG and its adjacent PGAM1 residues, for both the wild type system (upper panel) and the Y26-phospho system (bottom panel). The rows correspond to various hydrogen bonds formed. The columns correspond to time zones. 
using methods such as MM/GBSA [13-15, 28]. We extracted 5000 snapshots from the last 200 ns trajectory and used the data to calculate $\Delta G_{\text {bind }}$. The results are presented in Table 3 and Supplementary Figure 3. The binding free energy for the Y26-phospho system was lower than the wild type system, which indicated that the
2,3-BPG molecule bound to the PGAM1 protein more tightly in the Y26-phospho system than in the wild type system. To identify residues that are crucial to the binding, we calculated per-residue binding free energies $G_{\text {bind }}^{i}(i=$ $2,3, \ldots, 243)$ (Figure 7). One sees that the wild type system had only one primary contributor Arginine 10 (R10); while

Table 2: Properties of the hydrogen bonds formed between Y26 and its adjacent residues

(a) the wild type system

\begin{tabular}{|c|c|c|c|}
\hline H-bond & Occupancy (\%) & Distance $(\AA)$ & Angle ( $\left.{ }^{\circ}\right)$ \\
\hline None & N/A & N/A & N/A \\
\hline \multicolumn{4}{|c|}{ (b) the Y26-phospho system } \\
\hline H-bond & Occupancy $(\%)$ & Distance $(\AA)$ & Angle $\left(^{\circ}\right)$ \\
\hline Y26@O3P-N135@HD22 & 15.64 & 2.75 & 155.43 \\
\hline Y26@O3P-N135@HD21 & 8.66 & 2.85 & 160.23 \\
\hline Y26@O2P-N135@HD22 & 7.27 & 2.78 & 164.56 \\
\hline
\end{tabular}

(A)

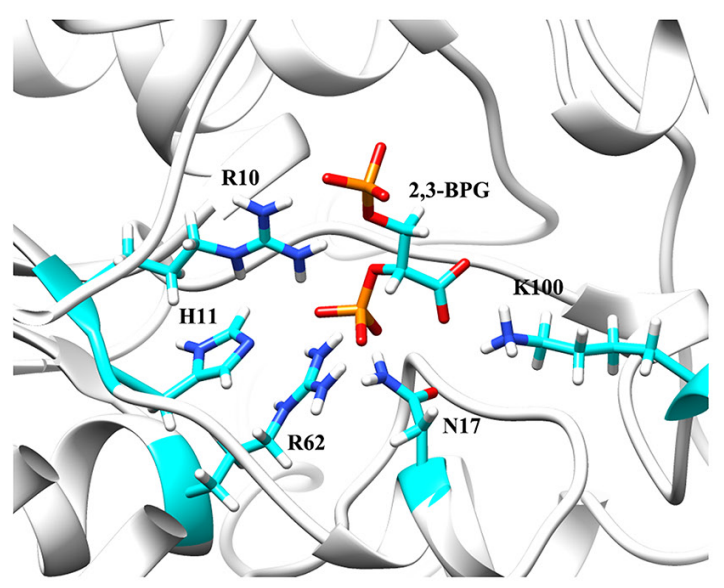

(B)

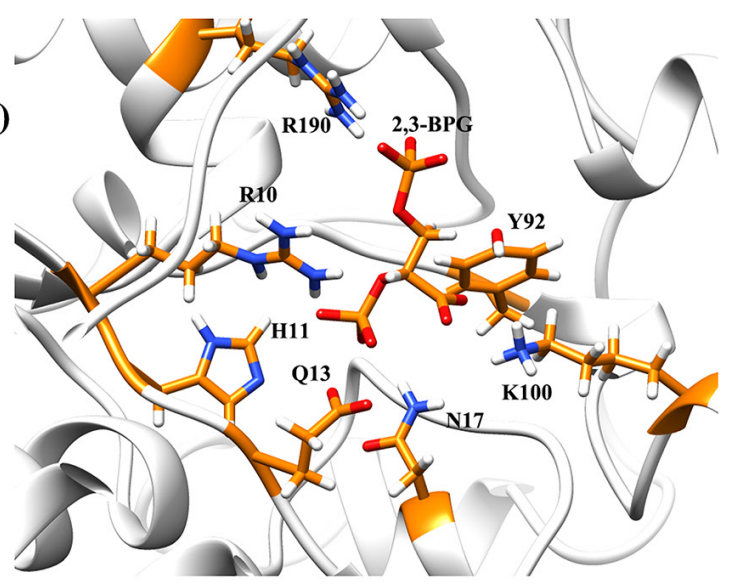

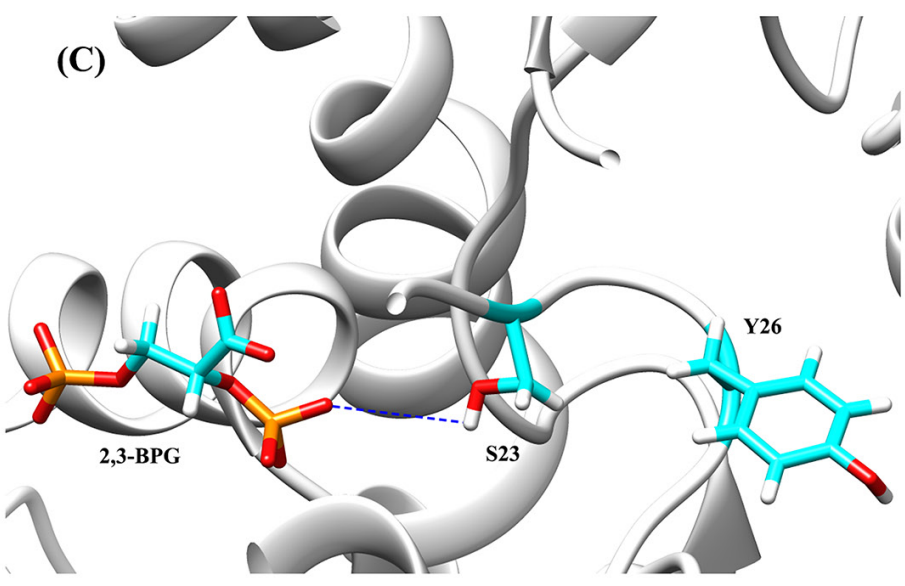

(D)

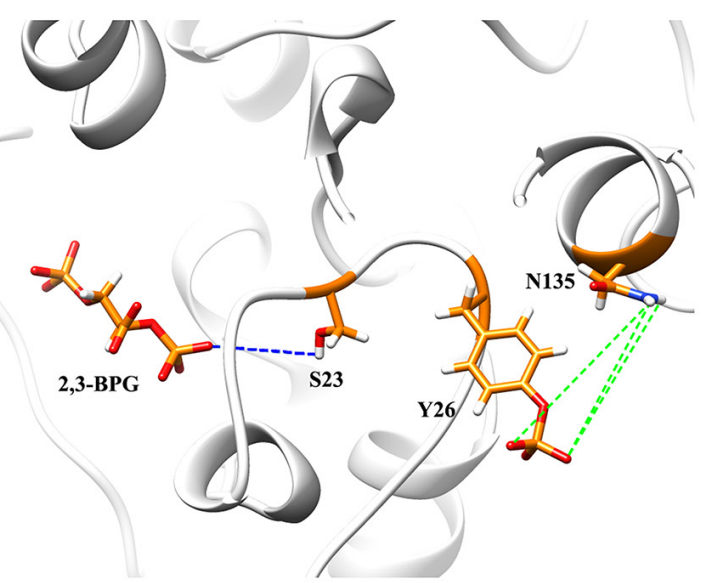

Figure 4: Structures of 2,3-BPG:PGAM1 complex for both the wild type system $A$ and $C$. and the Y26-phospho system B and D. 2,3-BPG, Y26, and their surrounding crucial residues are in stick representation. (A and B) 2,3-BPG is surrounded by a couple of ionic residues. (C) In the wild type system, the desired hydrogen bond 244@O15-S23@HG (the dashed blue line) is not formed due to the bad orientation between 2,3-BPG and S23. (D) In the Y26-phospho system, hydrogen bond 244@O15-S23@HG (the dashed blue line) is well formed, due to the three hydrogen bonds formed between the phosphorylated Y26 and N135 (the dashed green lines). 


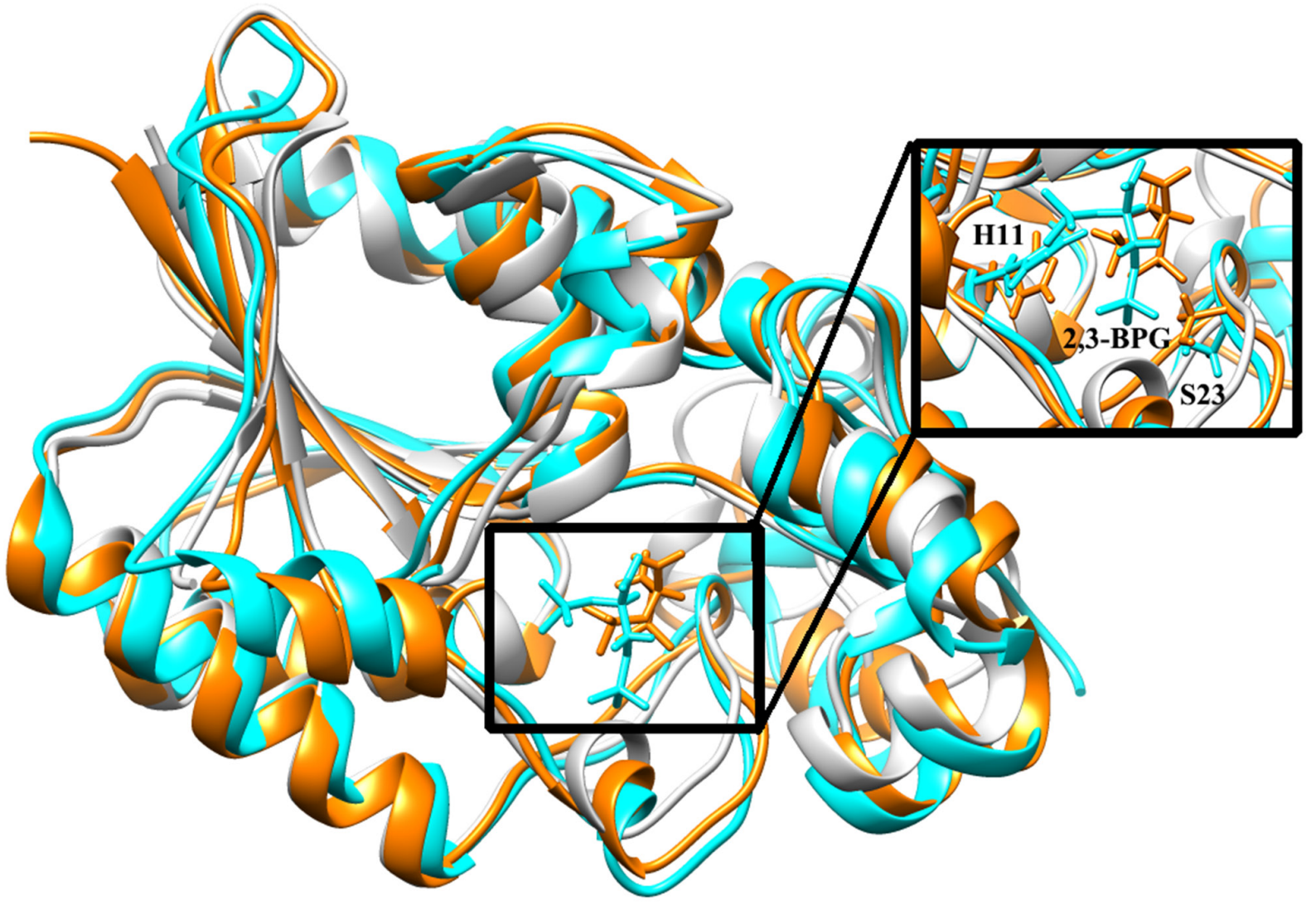

Figure 5: Superposition of $S_{3} w t$ (cyan), $S_{0}$ phos (orange), and the crystal structure 3FDZ (light gray). 2,3-BPG and some crucial residues of PGAM1 are in stick representation. The binding region is amplified.
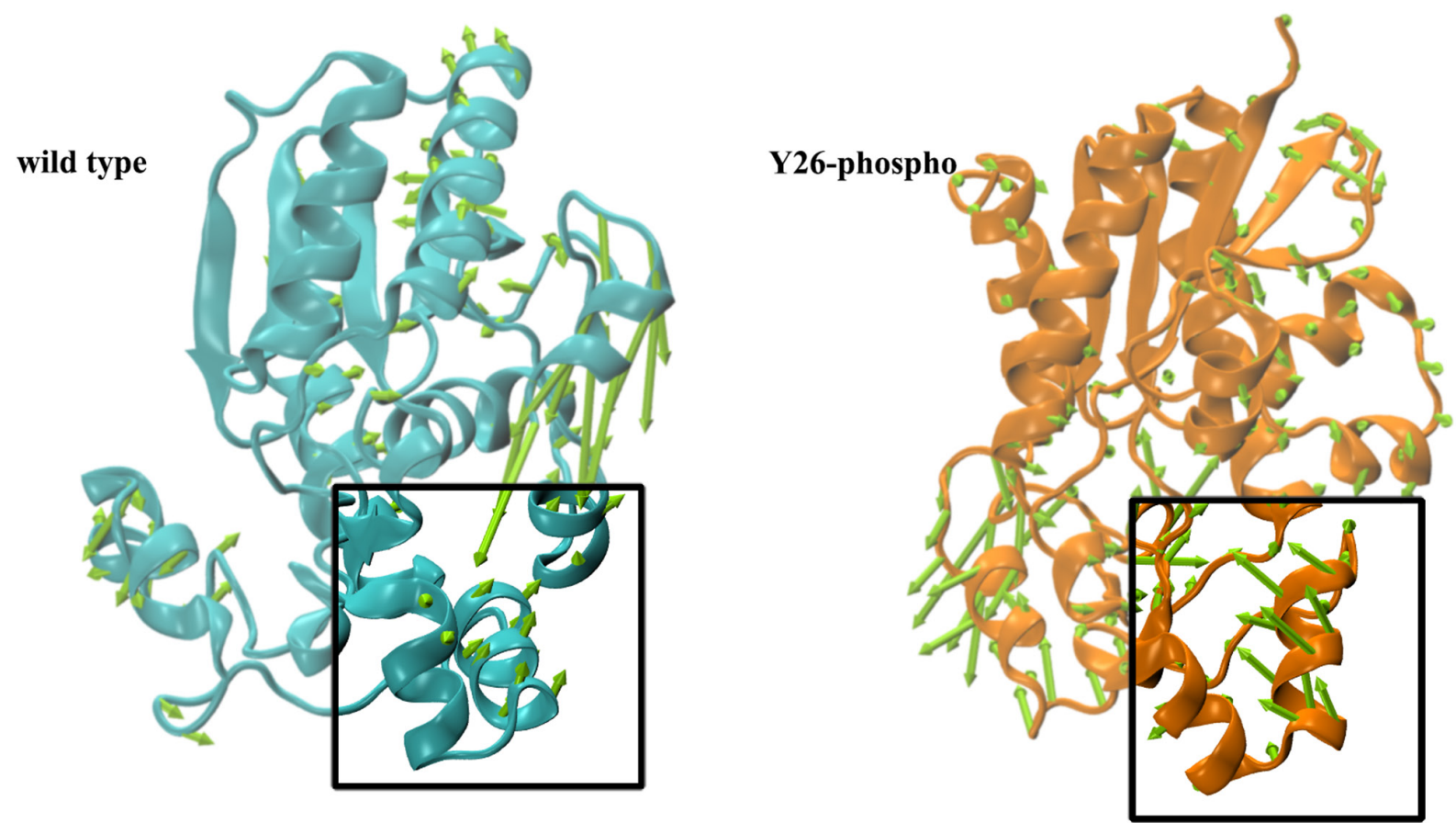

Figure 6: Porcupine plots for $\boldsymbol{S}_{3} \boldsymbol{w t}$ (cyan) and $\boldsymbol{S}_{\mathbf{0}}$ phos (orange). The conic arrows indicate directions of movement corresponding to the first principal component. The $\alpha$-helix region is boxed. 
Table 3: Total binding free energy $\left(\mathrm{kcal} \mathrm{mol}^{-1}\right)$ and its components in the 2,3-BPG:PGAM1 complex for the wild type and Y26-phospho systems

\begin{tabular}{|c|c|c|c|c|c|c|c|}
\hline System & $\Delta E_{\text {ele }}$ & $\Delta E_{v d w}$ & $\Delta \boldsymbol{G}_{G B}$ & $\Delta G_{S A S A}$ & $\Delta G_{M M / B G S A}{ }^{\mathrm{a}}$ & $-T \Delta S$ & $\Delta G_{\text {bind }}^{\mathrm{b}}$ \\
\hline wt & $-324.78 \pm 80.02$ & $-4.08 \pm 4.39$ & $333.38 \pm 74.20$ & $-3.32 \pm 0.28$ & $1.21 \pm 9.96$ & $18.60 \pm 5.07$ & $19.81 \pm 11.18$ \\
\hline Y26 & $-438.62 \pm 16.31$ & $-2.70 \pm 3.44$ & $441.46 \pm 12.73$ & $-2.97 \pm 0.046$ & $-2.82 \pm 5.25$ & $15.08 \pm 2.42$ & $13.25 \pm 5.78$ \\
\hline
\end{tabular}

the Y26-phospho system had three primary contributors: R10, K100, and Arginine 116 (R116). A residue far away from the binding pocket of 2,3-BPG, namely R191, was also worth noting. In the wild type system, R191 had much higher binding free energy than in the Y26-phospho system, which made it interact actively with 2,3-BPG and drag 2,3-BPG away from the binding pocket. This reduced the binding affinity of 2,3-BPG to the wild type PGAM1.

To understand why the binding was easy and tight in the Y26-phospho system but not in the wild type system, we examined individual energy and entropy terms in the binding free energy expression (Table 3 ). One sees that for both systems, the primary force favorable to the binding was the electrostatic force $\Delta E_{\text {ele }}$ : it was very negative $\left(-324.78 \mathrm{kcal} \cdot \mathrm{mol}^{-1}\right)$ in the wild type system and was even more negative $\left(-438.62 \mathrm{kcal} \cdot \mathrm{mol}^{-1}\right)$ in the $\mathrm{Y} 26$-phospho system. This is not strange, because 2,3-BPG is a highly negatively charged molecule, while its nearby PGAM1 residues are mostly positively charged residues such as $\mathrm{K} 100$ and R10, as revealed by the clustering analysis. This favorable electrostatic environment notwithstanding, the highly positive $\Delta G_{G B}=333.38 \mathrm{kcal} \cdot \mathrm{mol}^{-1}$ made the binding unfavorable, in the wild type system. In the Y26phospho system, electrostatic forces exerted by residue R10, K100, R116, S23, and N17 increased markedly (Figure 7), making the binding favorable.

The tightly bound 2,3-BPG might interact more actively with its surrounding residues, including K100 and R116, than in the wild type system. Note that K100 belongs to the $\alpha$-helix discovered in the cluster and PCA analyses. Therefore, the active interaction might bring $\mathrm{K} 100$ closer to 2,3-BPG, and further attracted the $\alpha$-helix moving towards 2,3-BPG (Figure 6).

\section{Y26 phosphorylation of PGAM1 enhanced glycolysis}

PGAM1 catalyzes the eighth step of glycolysis, namely the isomerization $3 \mathrm{PG} \rightarrow 2 \mathrm{PG}$ via the intermediate 2,3-BPG. Phosphorylation of PGAM1 at Y26 was hypothesized as an important mechanism to enhance glycolysis and consequently tumor growth [2].

To test this hypothesis, we had studied the second half reaction of the isomerization: $2,3-\mathrm{BPG} \rightarrow 2 \mathrm{PG}$. As mentioned above, these studies had been centered on the binding of 2,3-BPG to PGAM1, namely how Y26 phosphorylation stabilized the binding of 2,3-BPG to PGAM1. How about the subsequent events? Would Y26 phosphorylation help the production of 2PG, namely the transfer of the phosphoryl group from the $\mathrm{C}-3$ position of 2,3-BPG to H11 residue of PGAM1? The superimposition in Figure 5 had suggested that the distance and orientation between the donor and receptors are better in the Y26phospho system than in the wild type system. To test the intuition, we monitored the distance between P6 atom of 2,3-BPG and NE2 atom of H11 over the $900 \mathrm{~ns}$ simulation. We found that the distance fluctuated fiercely all over the simulation in the wild type system, with the largest distance even reaching $\sim 16 \AA$ (Figure 8A, black curve). In the Y26-phospho system, the fluctuation was much smaller (Figure 8A, red curve). At the end of the simulation, the distance in the Y26-phospho system was smaller than in the wild type system. These data suggested that the transfer of the phosphoryl group is much easier to occur in the Y26-phospho system, due to the overall closer distance between the donor and the receptor.

We then studied the effect of Y26 phosphorylation to the first half reaction of isomerization: $3 \mathrm{PG} \rightarrow 2,3-$ BPG, also catalyzed by PGAM1. To evaluate the transfer of the phosphoryl group from H11 of PGAM1 to 3PG (whereby 2,3-BPG is obtained), we monitored the distance between $\mathrm{P}$ atom of $\mathrm{H} 11$ and $\mathrm{O}$ atom around the $\mathrm{C}-2$ position of 3PG over the $900 \mathrm{~ns}$ simulation. We found that in the wild type system the distances fluctuated fiercely all over the simulation, with the highest value around $\sim 11$ $\AA$ (Figure $8 \mathrm{~B}$, black lines). In the Y26-phospho system, the fluctuation was much smaller (Figure $8 \mathrm{~B}$, red lines). The average values were $8.52 \AA$ and $6.34 \AA$ for the wild type and Y26-phospho systems, respectively. These data suggested that the phosphoryl group on H11 is much easier to transfer to the $\mathrm{C}-2$ position of $3 \mathrm{PG}$ with the aid of Y26 phosphorylation.

Finally, we calculated the binding free energy of 3PG:PGAM1 (the reactant complex form) and 2PG:PGAM1 (the product complex form) and presented the results in Tables 4 and 5, respectively. For both 3PG:PGAM1 and 2PG:PGAM1, Y26 phosphorylation significantly reduced the binding free energy. Therefore, the function of Y26 phosphorylation is consistent throughout the entire eighth step of glycolysis. It stabilized the binding of all the three (3PG, 2,3-BPG, 2PG) to PGAM1 and thus secured the isomerization. 


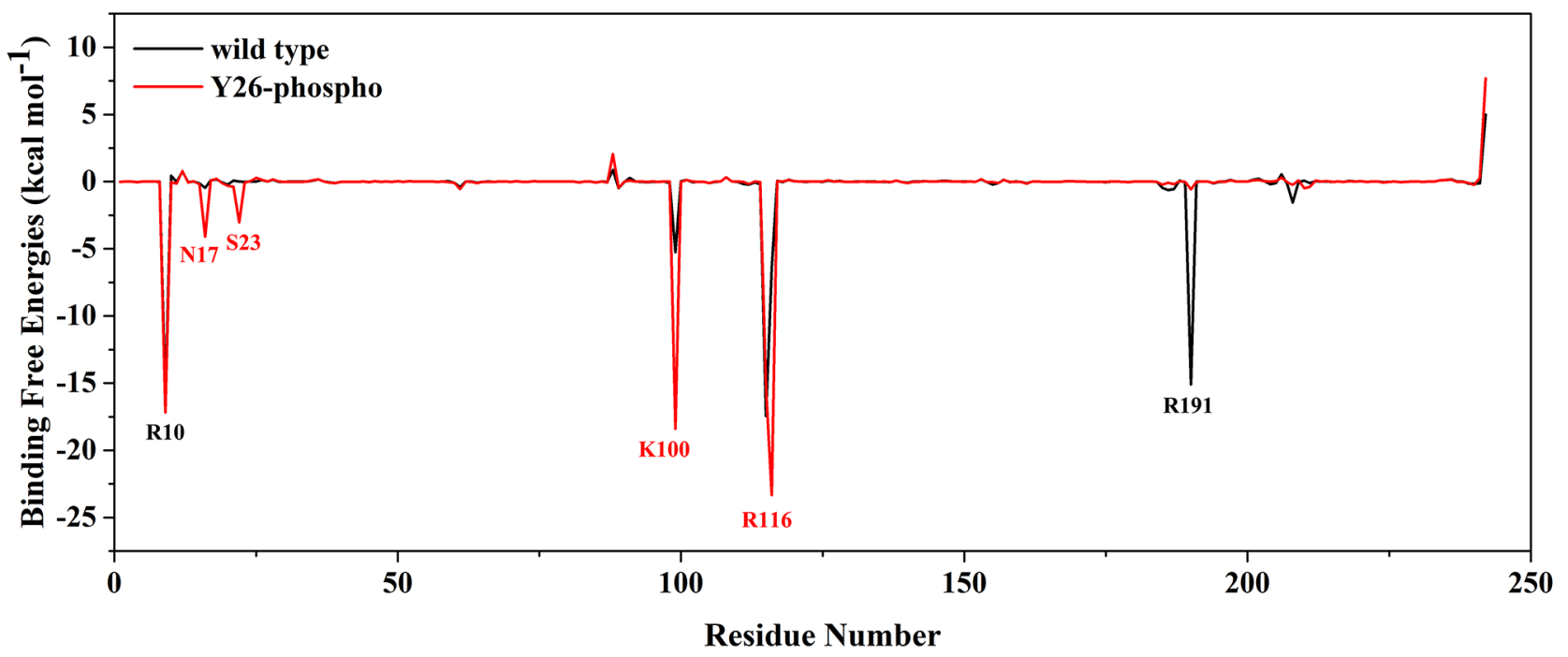

Figure 7: Per-residue binding free energies $G_{b i n d}^{i}(i=2,3, \ldots, 243)$ of the wild type system (black) and the Y26-phospho system (red). Residues with highly negative binding energy values are labeled.
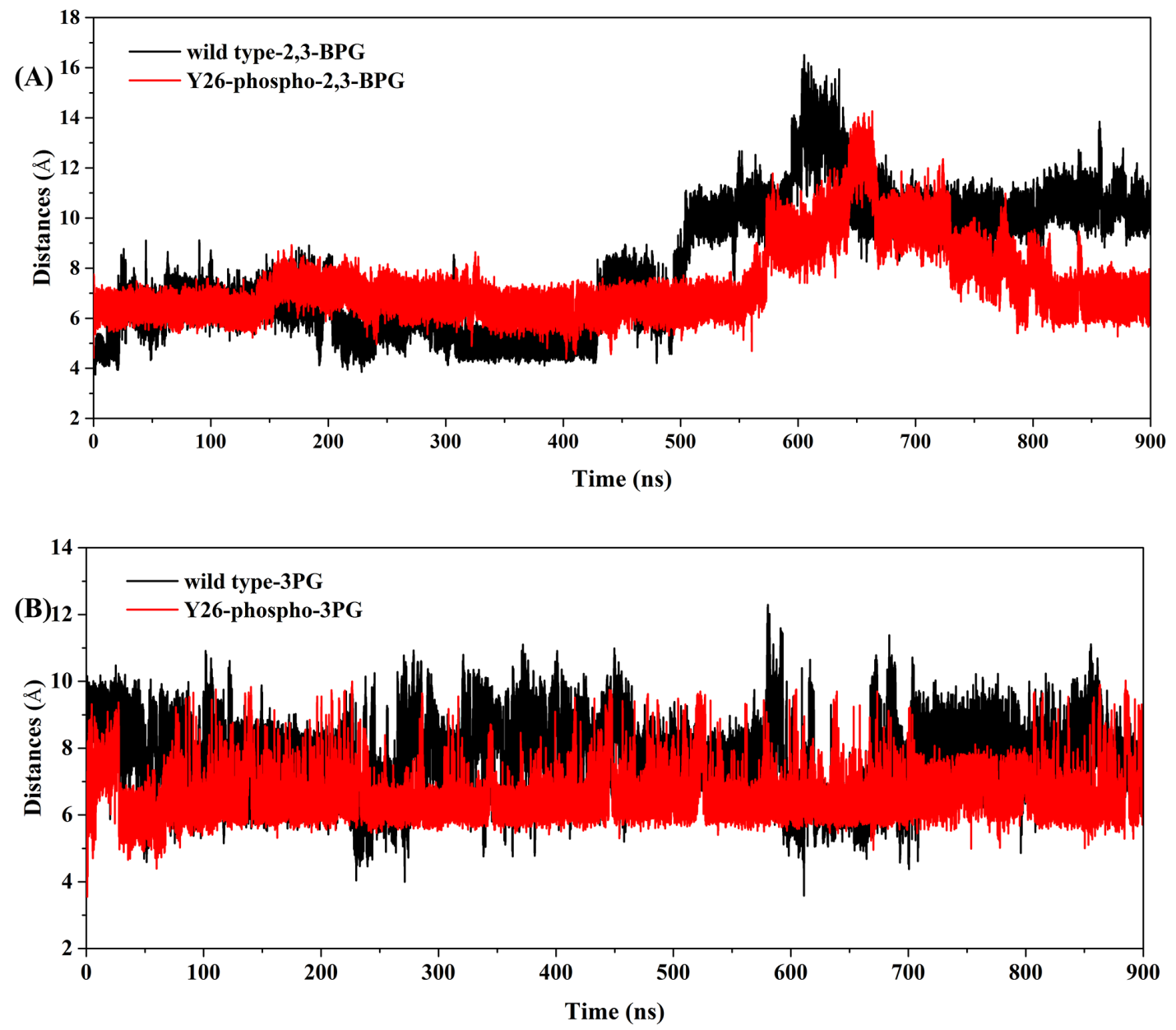

Figure 8: The distance plot over the 900 ns simulation time for both the wild type system (black lines) and the Y26phospho system (red lines). A. The distance between P6 atom of 2,3-BPG and NE2 atom of H11. B. The distance between O atom around the C-2 position of $3 \mathrm{PG}$ and $\mathrm{P}$ atom of $\mathrm{H} 11$. 
We then performed ASMD [29] simulations to study unbinding of the three ligands (2PG, 2,3-BPG and 3PG) from PGAM1. The detailed description of ASMD can be found in the Method section. The results were presented in Figure 9. One sees that all the three ligands were harder to dissociate from the PGAM1 protein in the Y26-phospho system than in the wild type system. That is, the energy barriers of the Y26-phospho system were all significantly higher than the wild type system. These results were consistent with the MM/GBSA free energy calculations. All the ligands bound more tightly in the Y26-phospho system than in the wild type system.

The isomerization manifested an "induced fit" characteristic of binding. That is, the binding affinity became increasingly greater as the substrate changed from $3 \mathrm{PG}$ to $2,3-\mathrm{BPG}$ and then to $2 \mathrm{PG}$, because $\Delta G_{\text {bind:3PG }}$ $>\Delta G_{\text {bind:2,3-BPG }}>\Delta G_{\text {bind:2PG }}$ (Tables 4 and 5). The order was followed by both the wild type and Y26-phospho systems.

\section{CONCLUSION}

As a crucial pathway of cellular metabolism, glycolysis is almost always accelerated in cancer cells to produce a vast amount of energy to sustain their rapid growth and division. Glycolytic enzymes are therefore often upregulated in cancer cells, including PGAM1, the enzyme catalyzing the eighth step of glycolysis. Hitosugi et al. hypothesized that the phosphorylation of Y26 residue of PGAM1 greatly enhances PGAM1 activity and contributes to carcinogenesis.

To test the hypothesis, we constructed two in silico PGAM1 systems with Y26 either dephosphorylated (the wild type system) or phosphorylated (the Y26-phospho system). We then compared the two systems in terms of structural stability, hydrogen bond formation, binding free energy, etc, by MD simulations. We found that Y26 phosphorylation enhances the binding of PGAM1 to its substrates through generating electrostatic environment

Table 4: Total binding free energy $\left(\mathrm{kcal} \mathrm{mol}^{-1}\right)$ and its components in the 3PG:PGAM1 complex for the wild type and Y26-phospho systems

\begin{tabular}{lccccccc}
\hline System & $\Delta \boldsymbol{E}_{\text {ele }}$ & $\Delta \boldsymbol{E}_{v d w}$ & $\Delta \boldsymbol{G}_{G B}$ & $\Delta \boldsymbol{G}_{\boldsymbol{S A S A}}$ & $\Delta \boldsymbol{G}_{\boldsymbol{M M} / \boldsymbol{B G S A}}{ }^{\mathrm{a}}$ & $-\boldsymbol{T} \Delta \boldsymbol{S}$ & $\Delta \boldsymbol{G}_{\text {bind }}{ }^{\mathrm{b}}$ \\
\hline wt & $-129.63 \pm 36.76$ & $-9.70 \pm 3.68$ & $164.97 \pm 33.56$ & $-3.26 \pm 0.17$ & $22.39 \pm 6.44$ & $16.60 \pm 5.00$ & $38.99 \pm 8.15$ \\
Y26 & $-136.26 \pm 94.44$ & $-7.88 \pm 3.24$ & $161.95 .46 \pm 40.20$ & $-2.59 \pm 0.46$ & $15.23 \pm 11.20$ & $16.89 \pm 5.83$ & $32.12 \pm 12.63$ \\
\hline
\end{tabular}

a $\Delta G_{M M / P B S A}=\Delta E_{\text {ele }}+\Delta E_{v d w}+\Delta G_{G B}+\Delta G_{S A S A}$

${ }^{\mathrm{b}} \Delta G_{T O T}=\Delta G_{M M / G B S A}-T \Delta S$.

Table 5: Total binding free energy $\left(\mathrm{kcal} \mathrm{mol}^{-1}\right)$ and its components in the 2PG:PGAM1 complex for the wild type and Y26-phospho systems

\begin{tabular}{|c|c|c|c|c|c|c|c|}
\hline System & $\Delta E_{\text {ele }}$ & $\Delta E_{v d w}$ & $\Delta \boldsymbol{G}_{G B}$ & $\Delta G_{S A S A}$ & $\Delta G_{M M / B G S A}{ }^{\mathrm{a}}$ & $-T \Delta S$ & $\Delta G_{\text {bind }}^{\mathrm{b}}$ \\
\hline wt & $-241.54 \pm 51.55$ & $-3.34 \pm 3.95$ & $210.04 \pm 45.69$ & $-3.37 \pm 0.14$ & $-38.21 \pm 9.76$ & $18.33 \pm 6.11$ & $-19.88 \pm 11.51$ \\
\hline Y26 & $-202.55 \pm 28.12$ & $-3.00 \pm 4.06$ & $156.75 .46 \pm 25.36$ & $-3.54 \pm 0.087$ & $-52.35 \pm 9.20$ & $16.89 \pm 5.83$ & $-35.46 \pm 10.89$ \\
\hline
\end{tabular}
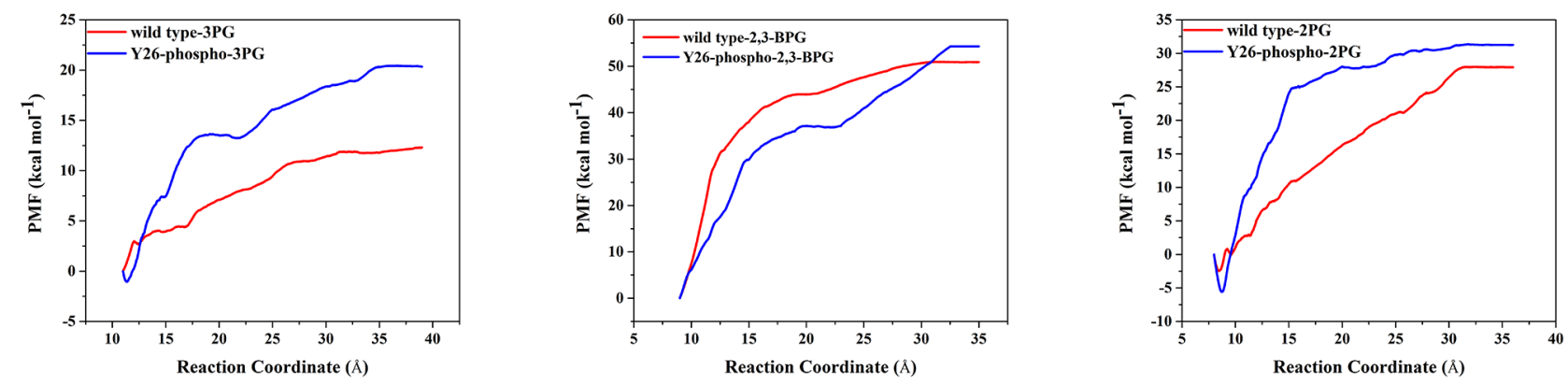

Figure 9: The PMF of pulling 3PG, 2,3-BPG, and 2PG from PGAM1. Red and blue lines are for the wild type and Y26-phospho systems, respectively. 
and structural features that are advantageous to the binding. For example, several Y26-phospho mediated hydrogen bonds facilitate the suitable orientation and distance between PGAM1 and its substrate. We also identified an $\alpha$-helix whose location and movement are both suitable for the substrate binding in the Y26-phospho system but not in the wild type system.

Our studies have revealed considerable atomistic details of PGAM1, its substrates, and their interactions, which may provide valuable insights into computeraided design of drugs that specifically target cancer cells. Through virtual screening of chemical libraries, for example, small drug molecules may be found that can greatly weaken or even block the binding between PGAM1 phos and its substrates, while having little effects on PGAM1 wt.

\section{MATERIALS AND METHODS}

\section{In silico models}

PGAM1 is a dimer of two identical monomers. According to the crystal structure 4GPZ (resolution: 1.65 $\AA$ ), the monomer consists of 242 amino acids (Alanine 2 to Methionine 243). We constructed an in silico model of the monomer by taking atomic coordinates from the crystal structure. This model was called PGAM1 wt. Because Y26 phosphorylation was the focus of the present study, we phosphorylated Y26 with Discovery Studio 4.0 Visualizer and created a new model PGAM1 phos. The protonation states of both models were carefully inspected based on the $\mathrm{H}++$ on-line server [30], with the results presented in Supplementary Table 1. According to the server calculation, all the histidine residues were set to HIE.

Our PGAM1 models were then docked with 2,3BPG. Because H11 in the unbound (apo) PGAM1 should be dephosphorylated but it was phosphorylated in the 4GPZ crystal structure, we used Discovery Studio 4.0 Visualizer to dephosphorylate H11 before the docking. The atomic coordinates of 2,3-BPG were obtained from the crystal structure 3FDZ [31], which was a 2,3-BPG:PGAM1 complex obtained from bacterium burkholderia pseudomallei. To replace bacterial PGAM1 with human PGAM1, we used the "superimposition method" $[14,32]$, which is effective in generating new protein/ligand complexes from old ones. Supplementary Figure 2 showed that 4GPZ and 3FDZ had high sequence identity (58.1\%) especially for the ligand binding residues. We first superimposed our PGAM1 model on 3FDZ and achieved a $0.80 \AA$ root-mean-square-deviation (RMSD) of superimposition. By removing all the PGAM1 atoms of 3FDZ, the desired human 2,3-BPG:PGAM1 complexes were created. The part of the complex centering around 2,3-BPG is shown for both the wild type system (Figure 4A) and the Y26-phospho system (Figure 4B). For both systems, 2,3-BPG is surrounded by a couple of ionic residues, which conforms with previous results that $3 \mathrm{PG}$ binds to an ionic pocket of PGAM1 [33]. This implies that the obtained 2,3-BPG:PGAM1 models are reasonable for MD simulations.

\section{Molecular dynamics simulation}

All the MD simulations were performed by using AMBER14 package [34] with ff14SB force field [35]. The force field parameters for the phosphorylated Y26 and 2,3-BPG were provided by Homeyer's phosphotyrosine parameter set [36] and the general AMBER force field (GAFF) [37], respectively. All the missing hydrogen atoms of PGAM1 were added by the LEaP module. Sodium ions were added by using coulomb potential grid to neutralize the whole system. The two systems were then respectively solvated in an octahedral periodic box, with TIP3P water model [38]. The distance between the outermost protein atoms and the walls of the simulation box was set to be $8.0 \AA$. With $8 \mathrm{Na}^{+}$and 8116 water molecules added, the wild type system had 28251 atoms in total. With $10 \mathrm{Na}^{+}$and 8271 water molecules added, the Y26-phospho system had 28721 atoms in total. The following procedures were applied to each of the two systems.

- Minimization Energy Minimization was performed to obtain a low-energy starting conformation for the subsequent MD simulations. A total of 10000 steps of Minimization were performed: 4000 of steepest descent followed by 6000 of conjugate gradient. The whole system (including the protein, the ligand, $\mathrm{Na}^{+}$, and the water molecules) was first minimized, followed by minimization on the protein and ligand only.

- Heating The system was then heated under NVT conditions (canonical ensemble) from 0 to $300 \mathrm{~K}$ for 300 ps, with the Langevin thermostat applied. The force constant for the harmonic restraint was set to be $10.0 \mathrm{kcal}$ $\mathrm{mol}^{-1} \AA^{-2}$.

- Equilibration The system was then equilibrated for $10 \mathrm{~ns}$ under NPT conditions (with constant pressure 1.0 bar). The relaxation time for barostat bath was set to be $2.0 \mathrm{ps}$.

- Simulation The system was finally simulated for $900 \mathrm{~ns}$ under NPT and periodic boundary conditions, with the time step set to be 2 fs. The long range electrostatics was handled by the particle-mesh Ewald (PME) method [39]. The cut-off value for short range interactions was set to be $10.0 \AA$. Bonds involving hydrogen atoms were constrained with SHAKE algorithm.

\section{Trajectory analysis}

A MD simulation usually generates a large bulk of data in the form of motion trajectories of all the atoms in the system. Valuable information can by yielded by 
analyzing the trajectories. In the present study, trajectories were analyzed with AmberTools 15.

- Hydrogen bond formation is sensitive to structural changes of biomolecules. In the present study, we used the following two criteria to judge whether or not a hydrogen bond is formed between an acceptor heavy atom $\mathrm{A}$, a donor hydrogen atom $\mathrm{H}$, and a donor heavy atom D. First, the distance between $\mathrm{A}$ and $\mathrm{H}$ is less than the distance cutoff $2.9 \AA$. Second, the A-H-D angle is greater than the angle cutoff $120^{\circ}$.

- Clustering. In the present study, a 900 ns MD simulation yielded 450000 snapshots (frames) of the trajectory. These snapshots were clustered into several groups, each containing molecular structures that are similar to each other. We used an average-linkage algorithm for clustering [40]. Each snapshot started as its own cluster; and the two closest clusters (judged by their distance) were merged in each iteration. The algorithm halted if the desired number $n$ (here $n=5$ ) of clusters had been obtained. The distance between clusters A and B was defined as the average of all the distances between a and $\mathrm{b}$, where a (b) is a snapshot in the cluster A (B). We picked five representative structures $S_{i}(i=0,1, \ldots, 4)$ from the five clusters, respectively. Note that $S_{0}$ was fixed to be the initial structure.

- Principal component analysis (PCA) can be used to distinguish few dominant motions of the system (the principal components) from many fluctuations that are of little functional importance [41]. In the present study, PCA was rendered by ProDy [42]; structural visualization and analysis were rendered by software VMD [43] and Chimera [44].

\section{Binding free energy calculations}

The free energy of receptor-ligand binding was calculated by the MM/GBSA method [13-15, 28]. To identify the most crucial residues of PGAM1 for the binding of 2,3-BPG, the total binding free energy was decomposed into contributions from individual residues $(i=2,3, \ldots, 243)$ :

$$
G_{\text {bind }}=\sum_{i=2}^{243} G_{\text {bind }}^{i}=\sum_{i=2} \sum_{j \neq i} G_{\text {bind }}^{i, j}
$$

where $G_{b i n d}^{i}$ are the per-residue contributions, $G_{\text {bind }}^{i, j}$ are the residue-pairwise interaction contributions. The calculations were rendered by the MMPBSA.py module [45] of AMBER14.

\section{Adaptive steered molecular dynamics}

Adaptive Steered Molecular Dynamics (ASMD) has been proved to be a powerful tool in studying dissociation of small ligands from proteins [29]. It was thus used to investigate the unbinding pathway of ligands from PGAM1. For an ASMD calculation, the overall reaction coordinate is divided into several stages. During each stage, we performed 40 Steered Molecular Dynamics (SMD) simulations following the approach described in [46], with the speed of the pulling force set to $1 \AA / \mathrm{ns}$. At the end of the stage, 40 trajectories had been generated, from which the potential of mean force (PMF) was calculated according to Jarzynski's equality [47]. The work done by the pulling force along each trajectory was calculated as well. To select the initial structure of the next stage from the ending conformations of all the 40 trajectories, we followed Hernandez and coworkers [29] by selecting the one whose corresponding trajectory had the work value closest to the PMF value. For determining the reaction coordinate, we applied tunnel analysis (see Supporting Information for details) implemented with the software package CAVER 3.0 and presented the results in Supplementary Figure 1. The reaction coordinate was determined to be the center of mass distance between the ligands (2PG, 2,3-BPG and 3PG) and Glu89. The initial coordinates of the ligands (2PG, 2,3-BPG and $3 \mathrm{PG}$ ) were selected from the representative structures of cluster analysis of the last 200ns of the total 900ns conventional MD simulation. At each stage of the ASMD simulation, 40 SMD simulation were performed, with the pulling speed set to $1 \AA / \mathrm{ns}$.

\section{CONFLICTS OF INTEREST}

The authors declare no conflicts of interest.

\section{GRANT SUPPORT}

This work was supported by National Program on Key Basic Research Project (2014CB910504), National Natural Science Foundation of China (61471186, 91529303, 91439103, 81471047), Startup Funds from Education Ministry of China, Shenzhen Municipal Research Funds (JCYJ20140417105816347), and Fundamental Research Grant of Southern University of Science and Technology (FRG-SUSTC1501A-38).

\section{REFERENCES}

1. Kroemer G, Pouyssegur J. Tumor cell metabolism: cancer's Achilles' heel. Cancer cell. 2008; 13:472-482.

2. Hitosugi T, Zhou L, Fan J, Elf S, Zhang L, Xie J, Wang Y, Gu T-L, Alečković M, LeRoy G. Tyr26 phosphorylation of PGAM1 provides a metabolic advantage to tumours by stabilizing the active conformation. Nat Commun. 2013; 4:1790

3. Britton H, Clarke J. The mechanism of the phosphoglycerate mutase reaction. Biochem J. 1969; 112:10P.

4. Voet D, Voet JG, Pratt CW. (1999). Fundamental of biochemistry. 
5. Ren F, Wu H, Lei Y, Zhang H, Liu R, Zhao Y, Chen X, Zeng D, Tong A, Chen L. Quantitative proteomics identification of phosphoglycerate mutase 1 as a novel therapeutic target in hepatocellular carcinoma. Mol Cancer. 2010; 9:1.

6. Liu L, Wang S, Zhang Q, Ding Y. Identification of potential genes/proteins regulated by Tiam1 in colorectal cancer by microarray analysis and proteome analysis. Cell Biol Int. 2008; 32:1215-1222.

7. Hitosugi T, Zhou L, Elf S, Fan J, Kang H-B, Seo JH, Shan C, Dai Q, Zhang L, Xie J. Phosphoglycerate mutase 1 coordinates glycolysis and biosynthesis to promote tumor growth. Cancer cell. 2012; 22:585-600.

8. Corcoran CA, Huang Y, Sheikh MS. The regulation of energy generating metabolic pathways by $\mathrm{p} 53$. Cancer Biol Ther. 2006; 5:1610-1613.

9. Tennant DA, Durán RV, Gottlieb E. Targeting metabolic transformation for cancer therapy. Nat Rev Cancer. 2010; 10:267-277.

10. Tennant DA, Durán RV, Boulahbel H, Gottlieb E. Metabolic transformation in cancer. Carcinogenesis. 2009; 30:1269-1280.

11. Aguirre-Alvarado C, Segura-Cabrera A, Velázquez-Quesada I, Hernández-Esquivel MA, García-Pérez CA, GuerreroRodríguez SL, Ruiz-Moreno AJ, Rodríguez-Moreno A, Pérez-Tapia SM, Velasco-Velázquez MA. Virtual screeningdriven repositioning of etoposide as CD44 antagonist in breast cancer cells. Oncotarget. 2016; 7:23772-23784. doi: 10.18632/oncotarget.8180.

12. Kuban-Jankowska A, Sahu K, Gorska M, Tuszynski J, Wozniak M. Chicoric acid binds to two sites and decreases the activity of the YopH bacterial virulence factor. Oncotarget. 2016; 7:2229-2238. doi: 10.18632/oncotarget.6812.

13. Wang Y, Zheng Q-C, Kong C-P, Tian Y, Zhan J, Zhang J-L, Zhang H-X. Heparin makes differences: a molecular dynamics simulation study on the human BII-tryptase monomer. Mol Biosyst. 2015; 11:252-261.

14. Wang Y, Zheng Q-C, Zhang J-L, Cui Y-L, Xue Q, Zhang H-X. Highlighting a $\pi-\pi$ interaction: a protein modeling and molecular dynamics simulation study on Anopheles gambiae glutathione S-transferase 1-2. J Mol Model. 2013; 19:5213-5223.

15. Wang Y, Zheng Q, Zhang J, Xie M, Zhan J, Zhang H. How mutations affecting the ligand-receptor interactions: a combined MD and QM/MM calculation on CYP2E1 and its two mutants. Chem Res Chinese U. 2015; 31:1029-1038.

16. Chu X, Liu F, Maxwell BA, Wang Y, Suo Z, Wang H, Han W, Wang J. Dynamic conformational change regulates the protein-DNA recognition: an investigation on binding of a Y-family polymerase to its target DNA. PLOS Comput Biol. 2014; 10:e1003804.

17. Wang Y, Tang C, Wang E, Wang J. PolyUbiquitin chain linkage topology selects the functions from the underlying binding landscape. Plos Comput Biol. 2014; 10:e1003691.
18. Wang Y, Gan L, Wang E, Wang J. Exploring the dynamic functional landscape of adenylate kinase modulated by substrates. J Chem Theory Comput. 2012; 9:84-95.

19. Wang Y, Chu X, Longhi S, Roche P, Han W, Wang E, Wang J. Multiscaled exploration of coupled folding and binding of an intrinsically disordered molecular recognition element in measles virus nucleoprotein. Proceedings of the National Academy of Sciences. 2013; 110:E3743-E3752.

20. Wang Y, Tang C, Wang E, Wang J. Exploration of multistate conformational dynamics and underlying global functional landscape of maltose binding protein. PLoS Comput Biol. 2012; 8:e1002471.

21. Wang Y, Chu X, Suo Z, Wang E, Wang J. Multidomain protein solves the folding problem by multifunnel combined landscape: Theoretical investigation of a Y-family DNA polymerase. J Am Chem Soc. 2012; 134:13755-13764.

22. Chu X, Wang Y, Gan L, Bai Y, Han W, Wang E, Wang J. Importance of electrostatic interactions in the association of intrinsically disordered histone chaperone $\mathrm{Chz} 1$ and histone H2A. Z-H2B. PLoS Comput Biol. 2012; 8:e1002608.

23. Wang J, Wang Y, Chu X, Hagen SJ, Han W, Wang E. Multi-scaled explorations of binding-induced folding of intrinsically disordered protein inhibitor IA3 to its target enzyme. PLoS Comput Biol. 2011; 7:e1001118.

24. Lu Q, Wang J. Single molecule conformational dynamics of adenylate kinase: energy landscape, structural correlations, and transition state ensembles. J Am Chem Soc. 2008; 130:4772-4783.

25. Lu Q, Lu HP, Wang J. Exploring the mechanism of flexible biomolecular recognition with single molecule dynamics. Phys Rev Lett. 2007; 98:128105.

26. Wang J, Lu Q, Lu HP. Single-molecule dynamics reveals cooperative binding-folding in protein recognition. PLoS Comput Biol. 2006; 2:e78.

27. Bond CS, White MF, Hunter WN. Mechanistic implications for Escherichia coli cofactor-dependent phosphoglycerate mutase based on the high-resolution crystal structure of a vanadate complex. J Mol Biol. 2002; 316:1071-1081.

28. Swanson J, Henchman RH, Mccammon JA. Revisiting free energy calculations: a theoretical connection to MM/ PBSA and direct calculation of the association free energy. Biophys J. 2004; 86:67.

29. Ozer G, Valeev EF, Quirk S, Hernandez R. Adaptive steered molecular dynamics of the long-distance unfolding of neuropeptide y. J Chem Theory Comput. 2010; 6:3026-3038.

30. Gordon JC, Myers JB, Folta T, Shoja V, Heath LS, Onufriev A. $\mathrm{H}++$ : a server for estimating pKas and adding missing hydrogens to macromolecules. Nucleic Acids Res. 2005; 33:W368-W371.

31. Davies DR, Staker BL, Abendroth JA, Edwards TE, Hartley R, Leonard J, Kim H, Rychel AL, Hewitt SN, Myler PJ. An ensemble of structures of Burkholderia pseudomallei 
2, 3-bisphosphoglycerate-dependent phosphoglycerate mutase. Acta Cryst F. 2011; 67:1044-1050.

32. Zhan J-Y, Zhang J-L, Wang Y, Li Y, Zhang H-X, Zheng Q-C. Exploring the interaction between human focal adhesion kinase and inhibitors: a molecular dynamic simulation and free energy calculations. Journal of Biomolecular Structure and Dynamics. 2016:1-16.

33. Crowhurst GS, Dalby AR, Isupov MN, Campbell JW, Littlechild JA. Structure of a phosphoglycerate mutase: 3-phosphoglyceric acid complex at 1.7 A. Acta Crystallogr, Sect D: Biol Crystallogr. 1999; 55:1822-1826.

34. Case D, Babin V, Berryman J, Betz R, Cai Q, Cerutti D, Cheatham Iii T, Darden T, Duke R, Gohlke H. (2014). Amber 14

35. Maier JA, Martinez C, Kasavajhala K, Wickstrom L, Hauser KE, Simmerling C. ff14SB: improving the accuracy of protein side chain and backbone parameters from ff99SB. J Chem Theory Comput. 2015; 11:3696-3713.

36. Homeyer N, Horn AH, Lanig H, Sticht H. AMBER forcefield parameters for phosphorylated amino acids in different protonation states: phosphoserine, phosphothreonine, phosphotyrosine, and phosphohistidine. J Mol Model. 2006; 12:281-289.

37. Wang J, Wolf RM, Caldwell JW, Kollman PA, Case DA. Development and testing of a general amber force field. J Comput Chem. 2004; 25:1157-1174.

38. Jorgensen WL, Chandrasekhar J, Madura JD, Impey RW, Klein ML. Comparison of simple potential functions for simulating liquid water. J Chem Phys. 1983; 79:926-935.
39. Darden TA, York DM, Pedersen LG. Particle mesh Ewald: An $\mathrm{N} \log (\mathrm{N})$ method for Ewald sums in large systems. J Chem Phys. 1993; 98:10089-10092.

40. Shao J, Tanner SW, Thompson N, Cheatham TE. Clustering Molecular Dynamics Trajectories: 1. Characterizing the Performance of Different Clustering Algorithms. J Chem Theory Comput. 2007; 3:2312-2334.

41. Amadei A, Linssen ABM, Berendsen HJC. Essential Dynamics of Proteins. Proteins. 1993; 17:412-425.

42. Bakan A, Meireles L, Bahar I. ProDy: Protein Dynamics Inferred from Theory and Experiments. Bioinformatics. $2011 ; 27: 1575-1577$.

43. Humphrey W, Dalke A, Schulten K. VMD-Visual Molecular Dynamics. J Mol Graph Model. 1996; 14:33-38.

44. Pettersen EF, Goddard TD, Huang CC, Couch GS, Greenblatt DM, Meng EC, Ferrin TE. UCSF Chimera-A visualization system for exploratory research and analysis. J Comput Chem. 2004; 25:1605.

45. Miller BR, Mcgee TD, Swails J, Homeyer N, Gohlke H, Roitberg AE. MMPBSA.py: An Efficient Program for EndState Free Energy Calculations. J Chem Theory Comput. 2012.

46. Bureau HR, Hershkovits E, Quirk S, Hernandez R. Determining the Energetics of Small $\beta$-Sheet Peptides using Adaptive Steered Molecular Dynamics. J Chem Theory Comput. 2016; 12:2028-2037.

47. Jarzynski C. Nonequilibrium equality for free energy differences. Phys Rev Lett. 1997; 78:2690. 